\title{
AVALIAÇÃO FISICO-BIOQUÍMICA E DENSITOMÉTRICA DE COLAGENO DE CICHLA OCELLARIS COMO FONTE POTENCIAL DE PÉPTIDOS PARA FINS INDUSTRIAIS
}

\author{
Vagne de Melo Oliveira ${ }^{1}$, Thiago Pajeú Nascimento ${ }^{2}$, Caio Rodrigo Dias Assis ${ }^{3}$, Ranilson Souza Bezerra ${ }^{3}$, \\ Ana Lúcia Figueiredo Porto ${ }^{1}$ \\ ${ }^{1}$ Departamento de Morfologia e Fisiologia Animal, UFPE, \\ ${ }^{2}$ Laboratório de Enzimologia, UFPE \\ ${ }^{3}$ Laboratório de Imunopatologia Keizo Asami (LIKA), UFPE \\ *thiago_pajeu@hotmail.com
}

\begin{abstract}
INTRODUCÃO
O aumento da produção e do consumo de pescado está diretamente relacionado à necessidade de se viabilizar tecnologias para o reaproveitamento dos resíduos gerados pela indústria aquí cola. De acordo com o Ministério das Pescas e Aquicultura (MPA) (MPA 2011), o Brasil tem uma produção anual de peixes de cerca de 1,4 milhão de toneladas, sendo 9.304.400 toneladas correspondentes ao peacock bass Cichla ocellaris). Essa espécie é rica por possui os seus resíduos ricos em colágeno.

O colágeno é uma proteína fibrosa sendo a principal componente de tecidos conjuntivos em mamíferos e peixes, representando $30 \%$ do conteúdo de proteína total e $6 \%$ em peso do corpo humano, desempenhando diferentes funções, especialmente estruturais, uma vez que são o principal componente estrutural de ossos, cartilagens, pele, tendões, ligamentos, músculo liso, vasos sanguíneos, dentes, córneas, lâmina basal e outros órgãos de vertebrados (Chung e Uitto 2010). Ela possui propriedades biomateriais relevantes como ponto de fusão baixo, biocompatibilidade, alta porosidade, facilidade de combinação com outros materiais, abundância e baixo custo, tornam o colágeno desejado para engenharia de tecidos, ao mesmo tempo em que seu processamento simples, baixa antigenicidade e alta absorção pelo corpo o tornam adequado para aplicações médicas (Subhan et al., 2015; Ferraro et al., 2016).

A geração de peptídeos bioativos é feita através da hidró lise do colágeno de peixe, esses peptídeos possuem diversas aplicações médicas e biológicas, por isso é necessária à busca de novas fontes de colágeno, uma vez que eles participam de processos como a adesão celular em tecidos vasculares e cutâneos, moléculas/acoplamentos de compostos bioativos e micro e nanoencapsulação (Ferraro et al., 2016). Assim, o presente estudo tem como objetivo determinar as propriedades físico-químicas do colágeno da pele do peito alto (C. ocellaris).
\end{abstract}

\section{MATERIAIS E MÉTODOS}

O colágeno foi obtido da extração das amostras de pele de espécimes adultos de peacock bass $C$. ocellaris com comprimento total de $70,5 \pm 1,5 \mathrm{~cm}$ e pesando $4,3 \pm 0,5 \mathrm{~kg}$ de uma aldeia de pescadores na cidade de Petrolândia, Pernambuco, Brasil. A extração de colágeno solúvel em pepsina (PSC) foi preparado com base nos métodos de Nagai e Suzuki (2000), com algumas modificações. Os espectros de absorção de UV do colágeno foram realizados de acordo com Nalinanon et al. (2011), utilizando um espectrofotômetro GeneQuant 1300, em comprimentos de onda na faixa de 200-280 nm. Para isso, as amostras de colágeno foram dissolvidas em solução de ácido acético 0,5 M numa proporção de 1: $1000(\mathrm{p} / \mathrm{v})$ e depois colocadas em uma cubeta de quartzo.

A espectroscopia de infravermelho de transformação de Fourier (iS 10, Thermo-Nicolet Co., EUA) foi utilizada para analisar a estrutura molecular do colágeno isolado. Para isso, misturou-se 1 $\mathrm{mg}$ de amostra de colágeno liofilizado numa proporção de aproximadamente 1: $100 \mathrm{com}$ brometo de potássio seco $(\mathrm{KBr})$ (espectro puro) e prensado em grânulos sob condições secas. Os espectros infravermelhos foram registrados na faixa de 4000-650 $\mathrm{cm}^{-1}$ na resolução de $1 \mathrm{~cm}^{-1}$ para cada varredura $(\mathrm{n}=8)$. Os espectros resultantes foram analisados usando o software Origem 8.0 (OriginLab Corporation, Northampton, MA, EUA) (Sun et al., 2017). A SDS-PAGE foi realizada seguindo o método de Laemmli (1970).

A análise de densitometria óptica de bandas individuais em cada pista $(n=3)$ do gel de eletroforese SDS-PAGE foi realizada pelo software Image $J$ (National Institutes of Health, Bethesda, MD, EUA). $\mathrm{Na}$ análise, as bandas formaram picos que foram quantificados como área sob os picos - AUP e como densidade integrada de pixels - ID, que representa a soma dos valores de intensidade de pixel (nos gráficos de Intensidade de Pixel versus Distância ao longo da Pista) de acordo com Oliveira et al. (2017a). Esta quantificação de bandas permitiu comparar o PSC de C. ocellaris com outras espécies (através da análise de estudos pré vios na literatura) utilizando as seguintes proporções de subunidades: cadeias a1/cadeia a2 (a1/a2), cadeias de monômero dímero/ total $B /(\alpha 1+a 2)$, cadeias trimer $\gamma /$ monômero total $\gamma /(a$ $1+a 2)$ e cadeias reticuladas / cadeias monoméricas totais $\mathrm{HMC} /(\mathrm{a}$ $1+a 2)$ e $V M C /(a 1+a 2)$; onde a HMC refere-se a componentes reticulados de alto peso molecular de colágeno e VMC a componentes reticulados de colágeno de muito alto peso molecular.

\section{RESULTADOS E DISCUSSÃO}

As proteínas têm geralmente uma absorção UV máxima a $280 \mathrm{~nm}$ (Huang et al., 2011), enquanto que o colágeno do peacock bass $C$. ocellaris apresentou maior taxa de atividade a $211 \mathrm{~nm}$, provavelmente devido à presença do domínio do telopeptídeo intacto. A não detecção de absorbância na amplitude de 240 a 280 nm sugere que o colágeno por si só não contém aminoácidos aromá ticos, como a tirosina e a fenilalanina. Assim, a proteína em questã 
o é o colágeno do tipo I. Os resultados são semelhantes aos relatados para peixes de tambor vermelho Sciaenops ocellatus (Chen et al., 2016). Estudos anteriores, usando colágeno tipo I da pele de espécies de peixes pelo método PSC, relataram absorções maiores que 220, como por exemplo, para colágeno de Rachycentron canadum, $221 \mathrm{~nm}$ (Zeng et al., 2012), Diodon holocanthus, $230 \mathrm{~nm}$ (Huang et al., 2011) e nódulos ornamentais de Nemipterus hexodon, $230 \mathrm{~nm}$ (Nalinanon et al., 2011).

A espectroscopia FT-IR tem sido usada para investigar modificações na estrutura secundária de colágeno, tais como desnaturação, reticulação, auto-montagem (Liu et al., 2012; Nalinanon et al., 2011; Di et al., 2014; Veeruraj et al., 2015; Chen et al. 2016). 0 espectro FT-IR do colágeno mostrou os cinco picos característicos das ligações amida que são os marcadores mais comuns da conformação do esqueleto do polipeptídico. Estes picos correspondem aos aminoácidos (prolina e hidroxiprolina) das molé culas intactas de colágeno conhecidas como amida A, amida B, amida I, amida II e amida III. De acordo com Muyonga et al. (2004b) e Veeruraj et al. (2013), a banda Amide A está relacionada ao alongamento $\mathrm{N}-\mathrm{H}$ acoplado à ligação de hidrogênio. As vibrações do alongamento $\mathrm{N}-\mathrm{H}$ livre ocorrem entre 3400 e $3440 \mathrm{~cm}^{-1}$ e quando o número da onda é deslocado para uma menor freqüência ( $3300 \mathrm{~cm}^{-1}$ ) significa que um grupo $\mathrm{N}-\mathrm{H}$ na molécula de colágeno está participando de uma ligação de hidrogênio e ajudando a manter a estrutura tripla helicoidal (Muyonga et al., 2004b, Veeruraj et al., 2013). O número de onda observado para esta banda na amostra de C. de peacock $C$. ocellaris foi de $3276 \mathrm{~cm}^{-1}$, indicando uma mudanç a considerável para uma freqüência mais baixa e evidenciando a ligação de hidrogênio restante no peacock bass $C$. ocellaris PSC da pele.

A manutenção da tripla hélice também é confirmada pela presença de componentes de colágeno reticulado de alto peso molecular (HMC), o trimer y e o dímero $B$ nas análises SDS-PAGE e densitometria óptica. Para PSC, a banda amida B está relacionada ao estiramento assimétrico $\mathrm{CH}_{2} \sim 3079 \mathrm{~cm} " 1 \mathrm{e} \sim 2924 \mathrm{~cm} " 1$ (Veeruraj et al., 2013; Muyonga et al., 2004a; 2004b) e flexão associada ao alongamento simétrico $\mathrm{CH}_{2}$ em $\sim 2854 \mathrm{~cm}^{-1}$ (Zeng et al., 2012). Embora as mudanças na estrutura secundária de colá geno não sejam confirmadas para atribuir mudanças na faixa espectral da banda de amida B (Muyonga et al., 2004b), a freqüê ncia de alongamento $\mathrm{CH}$ foi proposta como outro marcador conformacional por Bykov et al. (2008). No peacock bass C. ocellaris PSC, o número de onda de $2930 \mathrm{~cm}^{-1}$ foi encontrado para o pico da amida $\mathrm{B}$, sugerindo alongamento $\mathrm{CH}_{2}$ assimétrico e sem flexão. A banda Amida I apresenta absorção típica (aqui, transmitâ ncia no eixo Y) na faixa de números de onda de 1600 a $1700 \mathrm{~cm}^{-1}$, que é apontada como a região de absorção/ transmitância da vibração de estiramento de ligação $C=0$ no esqueleto do polipeptí dio de proteínas confirmado para ser parte da estrutura secundária quando em combinação com ligação de hidrogênio. Neste trabalho, a absorção/ transmitância de lone I de C. ocellaris PSC apresentou um downshift $\left(1637 \mathrm{~cm}^{-1}\right)$ em relação a esse intervalo caracterí stico, resultando em um pico de banda de amida I mais próxima ao componente encontrado em torno de $1630 \mathrm{~cm}^{-1}$ que pode indicar uma diminuição na ordem molecular e um grau de desnaturação (Muyonga et al. 2004a; 2004b). No entanto, o valor no presente estudo para a banda de amida II $\left(1547 \mathrm{~cm}^{-1}\right)$ corrobora os achados da amida A sobre a manutenção da estrutura tripla helicoidal, uma vez que constitui uma mudança para menor freqüência em relação à vibração característica da banda de amida II que ocorre na faixa espectral de 1550 a $1600 \mathrm{~cm}-1$. Esta faixa está associada à flexão de $\mathrm{N}-\mathrm{H}$ acoplada ao alongamento de $\mathrm{C}-\mathrm{N}$ e tal downshift evidencia a formação de ligação de hidrogênio mantendo as subunidades a ligadas em uma parte significativa do material extraído.

A presença da banda amida III de colágeno representa o alongamento do $\mathrm{CN}$ combinado com a flexão de $\mathrm{NH}$ para formar motivos de folhas B (Veeruraj et al., 2013) e foi encontrado no presente trabalho a uma freqüência de $1240 \mathrm{~cm} " 1$. De acordo com Muyonga et al. (2004b), uma relação de absorção/transmissão de aproximadamente 1,0 entre os valores da amida III e o pico em torno de $1449 \mathrm{~cm}^{-1}$ corrobora que a hélice tripla foi mantida extensivamente. Aqui, esta relação foi encontrada em 0.931 confirmando os resultados da amida A e da amida II.

0 perfil SDS-PAGE mostrou que os produtos alvo ( $a 1$ e $a 2$ ) foram obtidos (Fig. 1A). No entanto, bandas de dímero de colágeno e trímero (cadeias $B$ e $\mathrm{\gamma}$ ) e componentes reticulados de fibrilas de colágeno (HMC) podem ser observadas no gel e no densitograma (Fig. 1B). Em geral, em comparação com o colágeno solubilizado com ácido (ASC), o PSC apresenta bandas de degradação reticuladas de alto MW, principalmente VMC (Ahmad e Benjakul 2010; Kittiphattanabawon et al. 2010a; 2010b). Estes reticulados representam a clivagem de fibrilas de colágeno em pontos diferentes da região do tetrapeptideo, que é comum na extração acidental de colágeno. A Pepsina tem a capacidade de separar as ligações cruzadas intermoleculares para produzir cadeias monomé ricas.
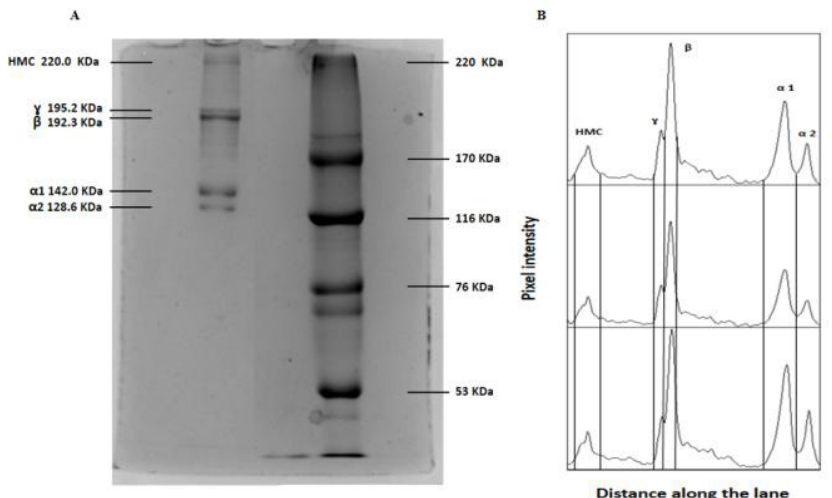

Figura 1. Padrão de proteína A-SDS-PAGE de colágeno tipo I pepsina solúvel extraído de Cichla ocellaris mostrando as cadeias de monómeros (a1 e a2), cadeia de dímero (B), cadeia de tríer (x) e componentes reticulados de alto peso molecular de colágeno (HMC); $B$ - Representação esquemática da amostragem de densidade integrada (ID) e área sob os picos (AUP) para bandas SDS-PAGE individuais de colágeno de pele tipo I de C. ocellaris. Identificando cada pico como a1, a2, B, y e HMC. Valores médios \pm SD para cada razão de cadeias a1 para cadeias a2 (a1 / a2), dímero B para cadeias monômeras totais B / (a1 + 2), trímero y para cadeias monômeras totais y / ( $(\alpha 1+a 2)$ e cruzadas As cadeias ligadas às cadeias monoméricas totais HMC / ( $(11+a 2)$ e VMC / ( 1 1 + a2) são mostradas na Tabela 1. HMC - componentes reticulados de alto peso de colágeno; VMC - componentes de colá geno reticulados de alta potência.

Algumas relações entre a quantidade de cadeias de subunidades e ligações cruzadas podem ser úteis quando se avalia a qualidade (e a eficiência, se o tempo de hidrólise for levado em consideração) da hidrólise. Essas relações são: relações de cadeia a 1 para cadeia a2 ( $\alpha 1 / \alpha 2$ ), dímero $B$ para cadeias monômeras totais $B /(a 1+a 2)$, trímero y para cadeias monômeras totais $y /(a 1+a 2)$ e reticuladas cadeias para as cadeias monoméricas totais $\mathrm{HMC} /$ ( $\mathrm{a} 1$ + a2) e VMC/(a1 + a2) (Nalinanon et al., 2011). Foi realizada uma comparação de tais proporções de géis de perfil de PSC de vários estudos e cujos valores foram calculados a partir dos densitogramas da Figura 2. 


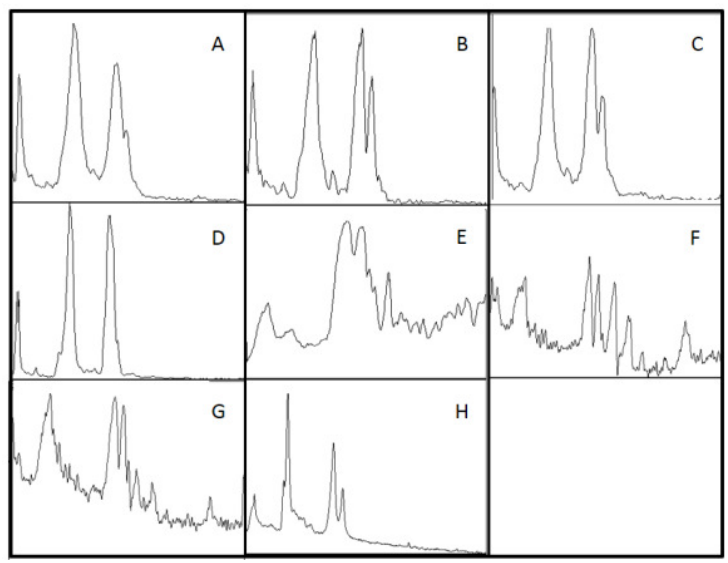

Figura 2. Densitogramas SDS-PAGE de colágeno de tipo I solubilizado com pepsina extraído da pele de: A - Aluterus monocerous submetido a pepsina porcina; B - Aluterus monocerous submetido a ATP (Thunnus alalunga pepsin); C - Aluterus monocerous submetido a ATP (Thunnus albacares pepsin); D - Chiloscyllium punctatum; E Rachycentron canadum; F - Ictalurus punctatus; G - Oreochromis niloticus; $\mathrm{H}$ - Os valores ID e AUP presentes foram estimados a partir desses densitogramas.

De acordo com a comparação, a fonte de pepsina não foi tão determinante para a qualidade de o produto de hidrólise, uma vez que a pepsina de peixe (três espécies de Scombridae) produziu mais subunidades a1 em relação a a2 comparando a pepsina porcina no estudo de Nalinanon et al. (2011) em 24h, enquanto ocorreu o contrário no trabalho de Ahmad e Benjakul (2010) em 48h. Talvez a enzima de peixes (espécies carnívoras) possa alcanç ar uma maior taxa de produção de a1 no início do processo e a enzima só poderia alcançá-la em um tempo de reação mais longo. Além disso, pode-se ver os valores da relação pepsina $B /(\alpha 1+a 2)$ variando de 0,10 a 1,22 , enquanto que a mesma proporção para pepsina de peixe variou de 0,88 a 1,21. A fonte de pepsina também não pareceu importante para as outras razões: $\gamma /(\alpha 1+a 2)$, $\mathrm{HMC} /(\mathrm{a} 1+\mathrm{a} 2)$ e VMC $/(\mathrm{a} 1+\mathrm{a} 2)$.

Por outro lado, o tempo de hidrólise exerceu um efeito significativo e aparentemente tendeu a reduzir a proporção de ligações HMC e principalmente VMC no PSC final. Os trabalhos que apresentaram ligações cruzadas do MVC foram aqueles que concluí ram a hidrólise em menos tempo (Nalinanon et al., 2011). A concentração de pepsina não afetou sozinhas as proporções. No entanto, em combinação com o tempo de hidrólise, maior concentração de pepsina $(0,5 \mathrm{U} / \mathrm{mg})$ no colágeno de pele de Nemipterus hexodon evidenciou maior conversão de VMC em HMC em relação à menor concentração desta enzima $(0,05 \mathrm{U} / \mathrm{mg})$ utilizada na hidrólise de Ictalurus punctatus e Oreochromis niloticus pele colágeno. Isto é confirmado no presente trabalho em que o VMC foi completamente convertido devido à alta concentraç ão de pepsina e maior tempo de hidrólise (Ahmad e Benjaku, 2010 ;Nalinanon et al., 2011; Kittiphattanabawon et al. 2010a; 2010b).

\section{CONCLUSÕES}

Com base nas análises de SDS-PAGE e densitometria ótica das subunidades monoméricas e reticências e as estruturas cellopeptídicas e helicoidais detectadas nos espectros de absorção UV e na espectroscopia FTIR; o material extraído pode ser caracterizado como colágeno tipo I, o que destaca sua importância para aplicações comerciais, sendo, portanto, potencialmente útil na produção de peptídeos de colágenos biologicamente ativos para setores alimentares, biomédicos e farmacêuticos.

\section{REFERÊNCIAS}

AHMAD, M., BENJAKU, S. Extraction and characterisation of pepsinsolubilised collagen from the skin of unicorn leatherjacket (Aluterus monocerous). Food Chem, 120: 817-824, 2010.

CHEN, J.; LI, L.; YI, R.; XU, N.; GAO, R.; HONG, B. Extraction and characterization of acid-soluble collagen from scales and skin of tilapia (Oreochromis niloticus). LWT - Food Sci Technol, 66: 453459, 2016.

CHUNG, H.J.; UITTO, J. Type VII collagen: the anchoring fibril protein at fault in dystrophic epidermolysis bullosa. Dermatol Clin, 28: 93-105, 2010.

FERRARO, V.; ANTON, M.; SANTÉ-LHOUTELLIER, V. The "sisters" ahelices of collagen, elastin and keratin recovered from animal byproducts: Functionality, bioactivity and trends of Application. Trends Food Sci Technol, 51: 65-75. 2016.

HUANG, Y.R.; SHIAU, C.Y.; CHEN, H.H.; HUANG, B.C. Isolation and characterization of acid and pepsin-solubilized collagens from the skin of balloon fish (Diodon holocanthus). Food Hydrocolloid, 25: 1507-1513, 2011.

KITTIPHATTANABAWON, P.; BENJAKUL, S.; VISESSANGUAN. W.; SHAHIDI. F. Isolation and characterization of collagen from the cartilages of brownbanded bamboo shark (Chiloscyllium punctatum) and blacktip shark (Carcharhinus limbatus). LWT Food sci. technol, 43: 792-800, 2010a.

KITTIPHATTANABAWON, P.; BENJAKUL, S.; VISESSANGUAN, W.; KISHIMURA, H.; SHAHIDI, F. Isolation and Characterisation of collagen from the skin of brownbanded bamboo shark (Chiloscyllium punctatum). Food Chem, 119: 1519-1526, $2010 \mathrm{~b}$.

LAEMMLI, U.K. Cleavage of structural proteins during the assembly of the head of bacteriophage T4. Nature, 227: 680-685, 1970.

LIU, D.; LIANG, L.;. REGENSTEIN, J.M.; ZHOU, P. Extraction and characterisation of pepsin-solubilised collagen from fins, scales, skins, bones and swim bladders of bighead carp (Hypophthalmichthys nobilis). Food Chem, 133: 1441-1448, 2012. MPA, Ministério da Pesca e Aquicultura. Boletim estatístico da pesca e aquicultura 2011. IOP Publishing PhysicsWeb. http://www.icmbio.gov.br/cepsul/images/stories/biblioteca/dow nload/estatistica/est_2011_bol_bra.pdf. Accessed 10 June 2016, 2011.

MUYONGA, J.H.; COLE, C.G.B.; DUODU, K.G. Characterization of acid soluble collagen from skins of young and adult Nile perch (Lates niloticus). Food Chem, 85: 815-89, 2004a.

MUYONGA, J.H.; COLE, C.G.B.; DUODU, K.G. Fourier transform infrared (FTIR) spectroscopic study of acid soluble collagen and gelatin from skins and bonés of young and adult Nile perch (Lates niloticus). Food Chem, 86: 325-332, 2004b.

NAGAI, T.; SUZUKI, N. Isolation of collagen from fish waste material-skin, bone and fins. Food Chem, 68: 277-281, 2000.

NALINANON, S.; BENJAKUL, S.; KISHIMURA, H.; OSAKO, K. Type I collagen from the skin of ornate threadfin bream (Nemipterushexodon): Characteristics and effect of pepsin hydrolysis. Food Chem, 125: 500-507, 2011.

OLIVEIRA, V.M.; ASSIS, C.R.D.; COSTA, H.M.S.; SILVA, R.P.F.; SANTOS, J.F.; CARVALHO JUNIOR, L.B.; BEZERRA, R.S. Aluminium sulfate exposure: A set of effects on hydrolases from brain, muscle and digestive tract of juvenile Nile tilapia (Oreochromis niloticus). Comp Biochem Physiol Pharmacol C, 191: 101-108, 2017a.

SUBHAN, F.; IKRAM, M.; SHEHZAD, A.; GHAFOOR, A. Marine collagen: an emerging player in biomedical applications. J Food Sci Technol, 52: 4703-4707, 2015.

SUN, L.; HOU, H.; LI, B.; ZHANG, Y. Characterization of acid- and pepsin-soluble collagen extracted from the skin of Nile tilapia (Oreochromis niloticus). Int J Biol Macromolec, 99: 8-14, 2017. VEERURAJ, A.; ARUMUGAM, M.; BALASUBRAMANIAN, T. Isolation and characterization thermostable collagen from eel-fish (Evenchelysmacrura). Process Biochem, 48: 1592-1602, 2013. 
VEERURAJ, A.; ARUMUGAM, M.; AJITHKUMAR, T.; BALASUBRAMANIAN, T. Isolation and characterization of collagen from the outer skin of squid (Doryteuthissinghalensis). Food Hydrocolloid, 43: 708-716, 2015.

ZENG, S.; YIN, J.; YANG, S.; ZHANG, C.; YANG, P.; WU, W. Structure and characteristics of acid and pepsin-solubilized collagens from the skin of cobia (Rachycentron canadum). Food Chem, 135: 1975-1984, 2012. 